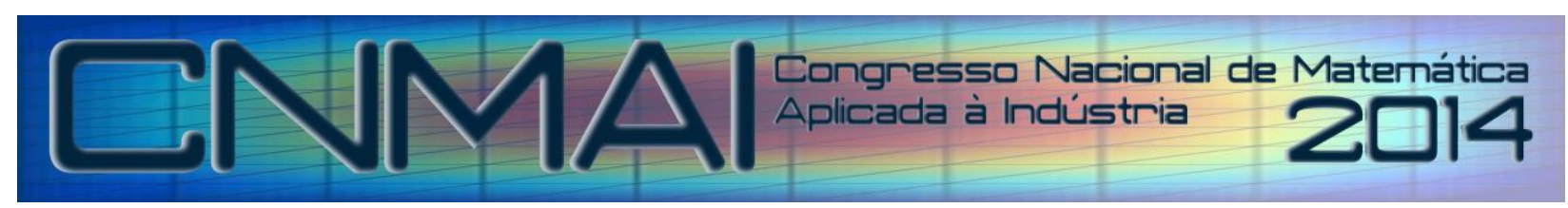

18 a 21 de novembro de 2014, Caldas Novas - Goiás

\title{
MODELAGEM MATEMÁTICA DO EFEITO DA CONDUÇÃO TÉRMICA NO PROCESSO DE AQUECIMENTO DE CABOS CONDUTORES
}

\author{
Rafael Augusto Magalhães Ferreira, r.ferreira102@ hotmail.com ${ }^{1}$ \\ Roberto Márcio de Andrade, roberto@demec.ufmg.br ${ }^{2}$ \\ ${ }^{1}$ Universidade Federal de Minas Gerais - Departamento de Engenharia Mecânica - Av. Antônio Carlos 6627, Belo \\ Horizonte - MG, Brasil. \\ ${ }^{2}$ Universidade Federal de Minas Gerais - Departamento de Engenharia Mecânica - Av. Antônio Carlos 6627, Belo \\ Horizonte - MG, Brasil.
}

\begin{abstract}
Resumo: Cabos condutores são elementos essenciais para o sistema elétrico de potência, em particular para o sistema de distribuição de energia. Nas subestações, devido à quantidade de equipamentos e subsistemas, muitas linhas energizadas são seccionadas e conectadas em diversos pontos, apresentando uma natural sobrelevação de temperatura nessas regiões, decorrente da geração de calor por Efeito Joule. A diferença de temperatura entre a região da conexão elétrica e dos cabos gera uma parcela de transferência de calor por condução, a qual pode ser modelada a partir de uma equação diferencial ordinária de segunda ordem, não homogênea e não linear. O presente trabalho tem por objetivo confrontar as soluções numérica e analítica do problema, avaliar a adequação das simplificações assumidas para o fenômeno físico e realizar a validação experimental através de ensaios de corrente. Os resultados apontam uma boa aproximação entre modelo e experimento e, sob o ponto de vista de monitoramento de cabos condutores, essa modelagem torna-se útil na identificação da distância, a partir do ponto de aquecimento, necessária para que a medição de temperatura no cabo não seja influenciada por efeitos de condução de calor axial; contribuindo para um aumento da confiabilidade dos registros térmicos e, consequentemente, do diagnóstico desse componente.
\end{abstract}

Palavras-chave: conectores elétricos, modelagem matemática, sistemas aletados, discretização de equações diferenciais.

\section{INTRODUÇÃO}

Durante um ciclo normal de operação em subestações ou nas linhas de transmissão de energia, o conjunto cabo/conector elétrico é submetido a diversas solicitações termomecânicas que incorrem em um processo natural de diminuição da vida útil de ambos componentes (Diniz, 2013). Esse desgaste ao longo do tempo de serviço está associado à oxidação de uma fração dos pontos de contato elétrico (Braunovic, 2006) e ao consequente aumento de densidade de corrente nos pontos restantes, ocasionando aumento de temperatura por Efeito Joule nessas regiões.

Nas proximidades das conexões, a descontinuidade física entre os cabos é fator determinante para que exista uma região preferencial de oxidação e, portanto, diminuição de pontos de contato elétrico. Desta forma, é recorrente a observação de anomalias térmicas nas proximidades das conexões, estas por sua vez apresentando temperaturas superiores ao restante dos cabos.

Essa diferença de temperatura entre a região da conexão elétrica e dos cabos gera uma parcela de transferência de calor por condução, que é tão significativa no balanço de energia quanto maior for essa variação. Ainda que não sejam projetados para esse fim, os cabos condutores nas proximidades de conexões defeituosas apresentam um comportamento similar a superfícies estendidas, e, em geral, esse efeito não pode ser desprezado na modelagem do processo de transferência de calor do conjunto. 


\section{MODELO MATEMÁTICO SIMPLIFICADO}

Existe uma extensa abordagem na literatura (Dewitt e Incropera, 2014; Çengel, 2009; Bejan, 1996) sobre o equacionamento de superfícies estendidas. Em geral, essas referências resolvem analiticamente a equação diferencial de segunda ordem associada a condução de calor unidirecional e apresentam soluções exatas para diferentes condições de contorno, dentre estas: temperatura prescrita, fluxo de calor prescrito, extremidade adiabática e aleta infinita.

No entanto, o presente problema apresenta uma particularidade que inviabiliza a aplicação de quaisquer dessas soluções. Como os cabos (aletas) apresentam resistência elétrica não desprezível e estão sujeitos à passagem de corrente, um termo de geração aparece na equação diferencial associada, demandando uma abordagem específica para essa condição.

De maneira simplificada, a geometria do problema pode ser representada segundo a Figura 1, a qual apresenta um desenho esquemático da conexão elétrica e um cabo de comprimento $L$. Em destaque, um elemento infinitesimal do cabo a partir do qual o equacionamento pode ser desenvolvido.

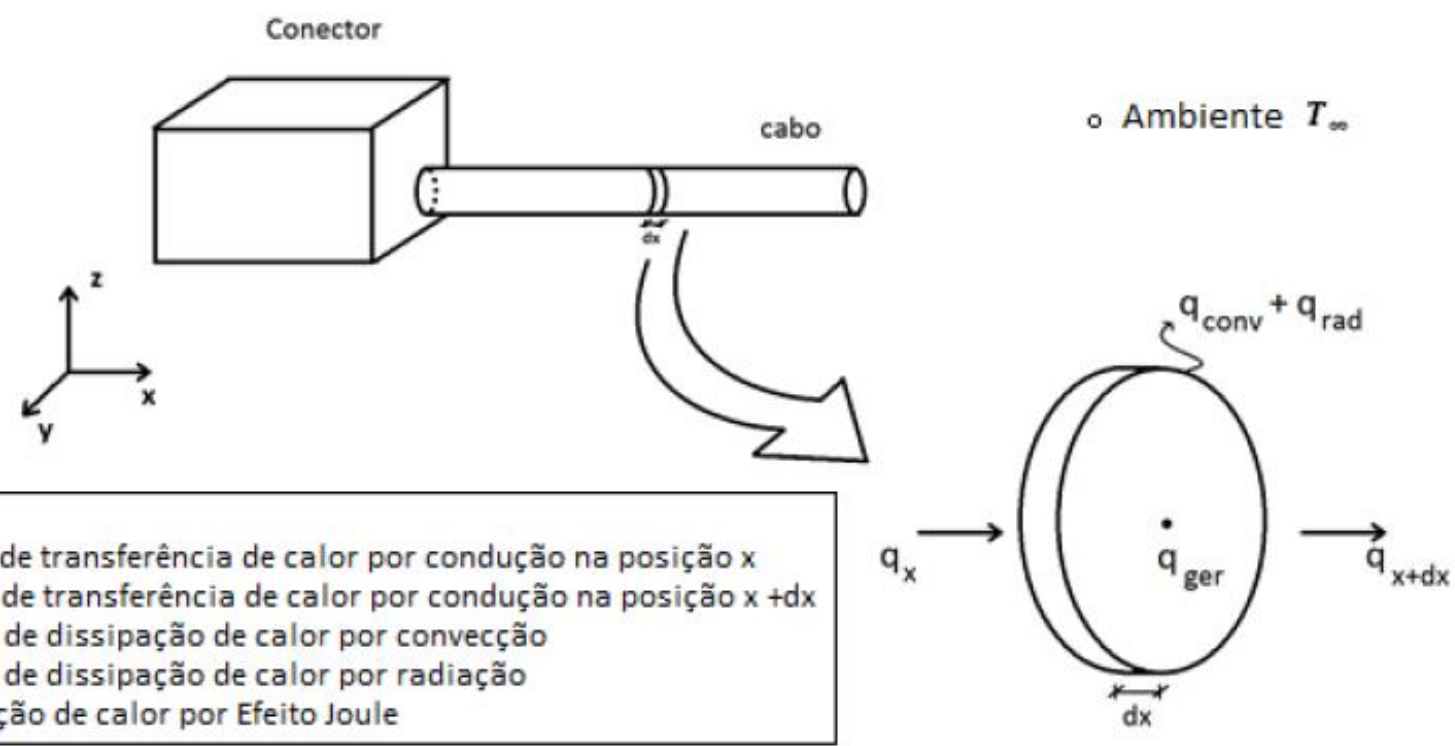

Figura 1. Simplificação geométrica do problema.

Aplicando o balanço de energia em regime permanente, as taxas de transferência de calor envolvidas se relacionam conforme a Eq. 1:

$$
\begin{gathered}
\sum q=0 \\
q_{x}-q_{x+d x}-\left(q_{c o n v}+q_{r a d}\right)+q_{g e r}=0
\end{gathered}
$$

Admitindo o cabo como uma aleta de seção reta constante e desconsiderando variações da condutividade térmica (k) do material em função da temperatura, tem-se:

$$
\begin{gathered}
k A_{s r} \frac{d^{2} T}{d x^{2}}+k \frac{d T d A_{s r}^{\top}}{d x d x}-h_{T}\left(T-T_{\infty}\right) \frac{A_{s u p}}{\Delta x}+\frac{\rho i^{2}}{A_{s r}}=0 \\
\frac{\boldsymbol{d}^{2} \boldsymbol{T}}{\boldsymbol{d} \boldsymbol{x}^{2}}-\frac{\boldsymbol{h}_{\boldsymbol{T}} \boldsymbol{P}}{\boldsymbol{k} \boldsymbol{A}_{\boldsymbol{s} r}}\left(\boldsymbol{T}-\boldsymbol{T}_{\infty}\right)+\frac{\boldsymbol{\rho} \boldsymbol{i}^{2}}{\boldsymbol{k} \boldsymbol{A}_{\boldsymbol{s r}}{ }^{2}}=\mathbf{0}
\end{gathered}
$$

Onde $i$ é a corrente aplicada, $A_{s r}$ e $P$ são a área e o perímetro da seção reta do cabo, respectivamente. A Eq. 4 é, portanto, a equação de governo da aleta para este problema. Trata-se de uma equação diferencial ordinária de segunda ordem, não homogênea e não linear. A não linearidade desta equação diferencial decorre do fato dos coeficientes $h_{T}$ (coeficiente total de dissipação) e $\rho$ (resistividade do material) serem função da temperatura. A aplicação de ferramentas matemáticas mais simples não permite a obtenção da solução analítica para equações nesse formato, a menos de uma simplificação para o caso de coeficientes constantes. Para fins de comparação, os resultados obtidos por essa aproximação serão confrontados com a solução numérica do problema. 


\subsection{Solução numérica}

Os $\mathrm{Na}$ Eq. 4, o temo da derivada segunda da temperatura em função da distancia ao longo do eixo $x$ pode ser aproximado, por diferenças centrais (Rocha, 1988), pela Eq. 5:

$$
\frac{d^{2} T}{d x^{2}}=\frac{T_{x_{i+1}}-2 T_{x_{i}}+T_{x_{i-1}}}{(\Delta x)^{2}}
$$

Onde os índices $i+1$ e $i-1$ se referem, respectivamente, aos pontos imediatamente à direita e à esquerda do $i$ ésimo nó da aleta discretizada. Obviamente, esta equação só vale para os nós internos do domínio $(0<x<L)$. As relações válidas nas extremidades $x=0$ e $x=L$ são definidas pelas condições de contorno do problema:

$$
\begin{gathered}
T(x=0)=T_{s, \text { conex }} \\
\left.\frac{d T}{d x}\right|_{x=L}=0
\end{gathered}
$$

As condições de contorno aplicadas definem em uma extremidade a temperatura da aleta como a temperatura do conector e, na extremidade oposta, que o fluxo de calor seja nulo. Essa segunda condição de extremidade isolada é válida para aletas longas, onde a temperatura da aleta se torna constante a partir de uma determinada posição $x=L$. O domínio físico tratado nesse modelo está, portanto, delimitado por essas duas extremidades.

Aplicando a Eq. 5 na Equação de governo da aleta (Eq. 4), tem-se:

$$
T_{x_{i+1}}=\frac{(\Delta x)^{2}}{k A_{s r}}\left[h_{T} P\left(T_{x_{i}}-T_{\infty}\right)-\frac{\rho i^{2}}{A_{s r}}\right]-T_{x_{i-1}}+2 T_{x_{i}}
$$

Essa solução é de fácil implementação e determina que a temperatura em um nó do domínio discretizado pode ser calculada a partir dos valores de temperatura dos dois pontos imediatamente anteriores. Todos os parâmetros dependentes da temperatura podem ser avaliados em função da temperatura do nó imediatamente anterior com um erro associado desprezível para pequenos valores de $\Delta x$ empregados.

É particularmente interessante calcular a quantidade de calor que cada um dos cabos retira do conector através do mecanismo de condução térmica. De posse do perfil de temperaturas na aleta, solução da equação diferencial estabelecida, esse cálculo pode ser realizado através da fórmula geral da condução (Lei de Fourier) aplicada à base da aleta, Eq. 9:

$$
q_{\text {cond, base }}=-k A_{s r} \frac{d T}{d x} \cong-k A_{s r} \frac{\left(T_{x_{1}}-T_{x_{0}}\right)}{\Delta x}
$$

Outra forma igualmente válida para determinação dessa taxa de transferência de calor é através de um balanço global de energia aplicado no cabo com comprimento suficiente para satisfazer a segunda condição de contorno definida como fluxo zero na extremidade oposta ao conector, conforme ilustrado na Figura 2.

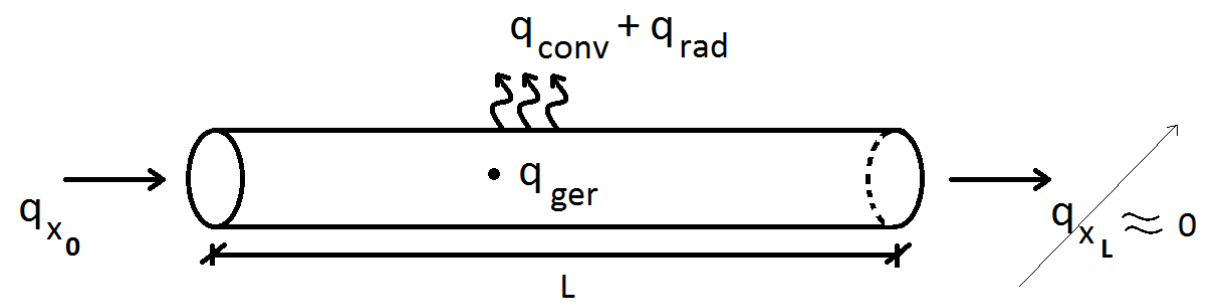

Figura 2. Determinação do fluxo de calor por condução segundo balanço global.

Aplicando o balanço de energia às taxas de transferência de calor acima apresentadas, tem-se:

$$
\begin{gathered}
\sum q=0 \\
q_{x_{0}}-q_{x_{L}}-\left(q_{c o n v}+q_{r a d}\right)+q_{g e r}=0 \\
\boldsymbol{q}_{x_{\mathbf{0}}}=\boldsymbol{h}_{\boldsymbol{T}}(\boldsymbol{P L})\left(\overline{\boldsymbol{T}}-\boldsymbol{T}_{\infty}\right)-\left(\frac{\boldsymbol{\rho} \boldsymbol{L}}{\boldsymbol{A}_{\boldsymbol{s r}}}\right) \boldsymbol{i}^{2}
\end{gathered}
$$


$\mathrm{Na}$ Eq.12, o coeficiente total de dissipação por convecção e radiação $\left(h_{T}\right)$ e a resistividade do material do cabo $(\rho)$ devem ser avaliados em função do valor médio de temperatura $(\bar{T})$ ao longo de todo o comprimento $L$ do cabo, resultado do perfil térmico obtido na solução da Eq. 4. Por definição, o cálculo do valor médio do perfil $T(x)$ envolve a integral dessa função no trecho considerado (Stewart, 2013) e, para fins práticos, representa o valor de temperatura para o qual a área do gráfico dentro do intervalo é equivalente ao produto $\bar{T}(b-a)$.

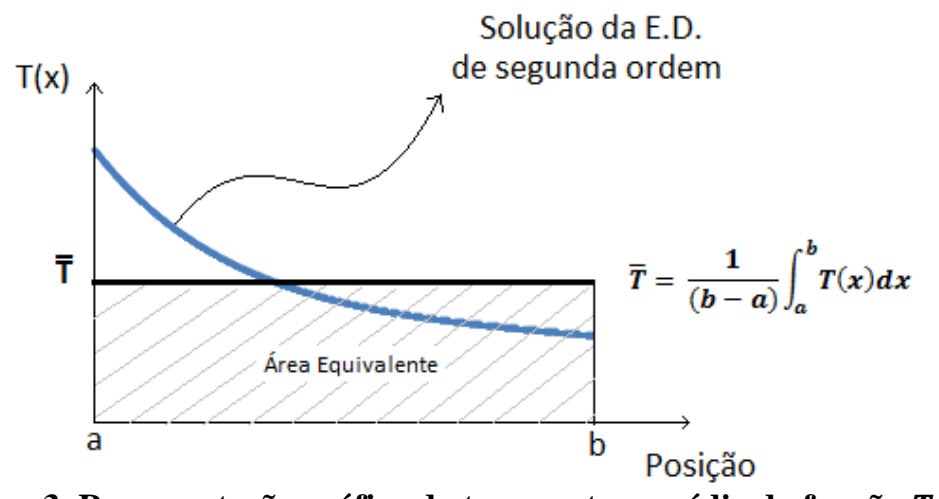

Figura 3. Representação gráfica da temperatura média da função $T(x)$.

Uma terceira e última maneira de determinar o fluxo de calor na base da aleta também passa pela aplicação do balanço de energia na aleta de comprimento $L$ que satisfaça a segunda condição de contorno, porém desta vez com uma abordagem através do fracionamento do domínio físico em $n$ elementos de dimensão reduzida, Figura 4.
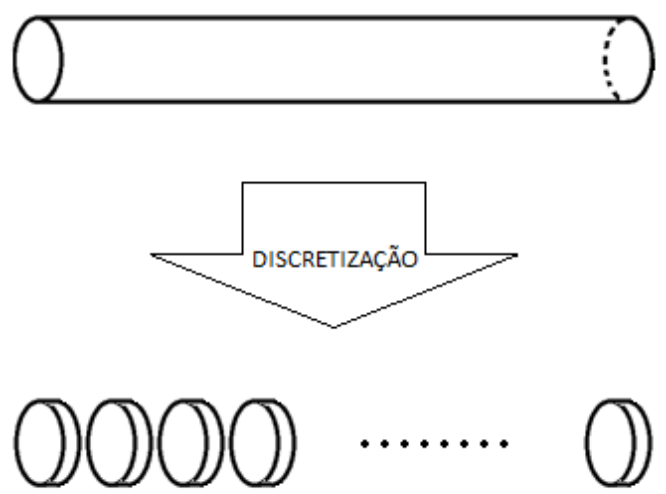

$\begin{array}{llll}1 & 2 & 3 & 4\end{array}$

$\mathrm{n}$

Figura 4. Fracionamento do domínio físico.

De posse do perfil de temperaturas obtido na solução da equação diferencial, a cada elemento definido fica associado uma taxa de geração de calor por Efeito Joule e uma taxa de dissipação de calor específica. Pelo balanço de energia, tem-se:

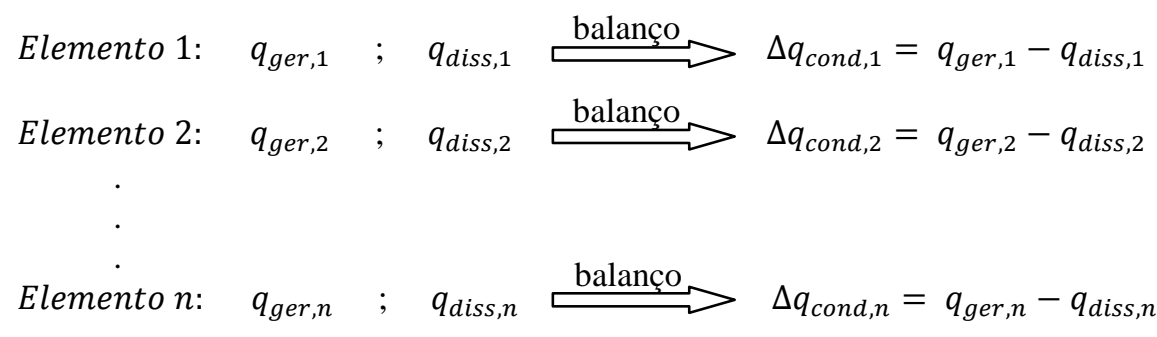

$$
q_{\text {cond }}=q_{x_{0}}-q_{x_{L}}^{\approx 0}=\sum_{i=1}^{n} q_{g e r, i}-\sum_{i=1}^{n} q_{d i s s, i}
$$

Esse método permite identificar o comportamento das taxas de transferência de calor ao longo do comprimento da aleta e, dessa forma, determinar para cada situação um comprimento limite, a partir do qual, os efeitos da condução axial de calor se tornam desprezíveis.

As soluções numéricas apresentadas nessa seção apesar de gerarem uma quantidade considerável de cálculos para obtenção do perfil térmico, são de simples implementação e permitem uma avaliação mais criteriosa do fenômeno físico ao levar em conta as variações espaciais dos coeficientes convectivo, de radiação, bem como das propriedades do material. 


\subsection{Solução analítica}

Para a determinação da solução analítica da Eq. 4, será aplicado o método de solução de equações diferenciais de segunda ordem, não homogêneas e coeficientes constantes, apesar da última afirmação não se verificar no presente caso devido à dependência de alguns termos da equação com a temperatura. Parte deste problema pode ser contornado expandindo a equação e explicitando a relação direta entre resistividade e temperatura, como se segue:

$$
\frac{d^{2} T}{d x^{2}}-\frac{h_{T} P}{k A_{s r}}\left(T-T_{\infty}\right)+\frac{\rho_{0}\left[1+\alpha\left(T-T_{\infty}\right)\right] i^{2}}{k A_{s r}^{2}}=0
$$

Generalizando, a Eq.14 pode ser reescrita como:

Onde,

$$
a T^{\prime \prime}+b T^{\prime}-c T=-g(x)
$$

$$
\begin{aligned}
& a=1 \\
& b=0 \\
& c=\frac{h_{T} P}{k A_{s r}}-\frac{\alpha \rho_{0}}{k A_{s r}{ }^{2}} i^{2}>0 \\
& g(x)=\left(\frac{h_{T} P}{k A_{s r}}-\frac{\alpha \rho_{0}}{k A_{s r}{ }^{2}} i^{2}\right) T_{\infty}+\frac{\rho_{0} i^{2}}{k A_{s r}{ }^{2}}=M>0
\end{aligned}
$$

Portanto:

$$
T^{\prime \prime}-c T=-M
$$

Equação característica:

$$
r^{2}-c=0 \Longrightarrow r= \pm \sqrt{c}
$$

Então a solução homogênea da Eq.14 será dada por:

$$
T_{h}(x)=c_{1} e^{\sqrt{c} x}+c_{2} e^{-\sqrt{c} x}
$$

Para a solução particular, utiliza-se o Método dos Coeficientes a Determinar (Santos, 2011), onde deve-se procurar uma solução do tipo $T_{p}(x)=x^{s}\left(A_{0}+\cdots+A_{n} x^{n}\right)$. Como $g(x)$ é uma constante, $s=0$ e então:

$$
\begin{gathered}
g(x)=-M=a_{0} \\
T_{p}(x)=x^{s}\left(A_{0}\right)=x^{0}\left(A_{0}\right)=A_{0}
\end{gathered}
$$

Logo:

$$
T_{p}^{\prime}(x)=T_{p}^{\prime \prime}(x)=0
$$

Substituindo na Eq. 20, encontra-se a solução particular da equação:

$$
\begin{gathered}
-c A_{0}=-M \\
A_{0}=M / c \quad \Longrightarrow \quad \boldsymbol{T}_{\boldsymbol{p}}(\boldsymbol{x})=\mathrm{M} / \mathrm{c}
\end{gathered}
$$

Pela teoria de Equações Diferenciais, a solução geral a ser obtida é a soma das soluções particular e homogênea previamente encontradas:

$$
\begin{gathered}
T(x)=T_{h}(x)+T_{p}(x) \\
\boldsymbol{T}(\boldsymbol{x})=\boldsymbol{c}_{\mathbf{1}} \boldsymbol{e}^{\sqrt{c} x}+\boldsymbol{c}_{\mathbf{2}} \boldsymbol{e}^{-\sqrt{c} x}+\boldsymbol{M} / \boldsymbol{c}
\end{gathered}
$$


Condições de contorno:

$$
\begin{aligned}
& T(x=0)=T_{s, \text { conex }} \quad \Longrightarrow \quad c_{1}+c_{2}+M / c=T_{s, \text { conex }}=T_{1} \\
& c_{1}=T_{1}-c_{2}-M / c \\
& \left.\frac{d T}{d x}\right|_{x=L}=0 \quad \longleftrightarrow \sqrt{c} c_{1} e^{\sqrt{c} L}-\sqrt{c} c_{2} e^{-\sqrt{c} L}=0 \\
& \left(T_{1}-c_{2}-M / c\right) e^{\sqrt{c} L}-c_{2} e^{-\sqrt{c} L}=0 \\
& c_{2}=\frac{e^{\sqrt{c} L}\left(T_{1}-M / c\right)}{e^{\sqrt{c} L}+e^{-\sqrt{c} L}}
\end{aligned}
$$

Portanto, as Equações (25) a (27) em combinação com as Equações (18) e (19) definem a solução analítica para o perfil de temperaturas da aleta com geração de calor. De posse da função distribuição de temperaturas, o fluxo de calor na base pode ser calculado de qualquer uma das três maneiras apresentadas na seção 2.1 .

\section{METODOLOGIA EXPERIMENTAL}

Foi montada uma estrutura de sustentação e energização de amostras de cabos nas imediações do galpão de Engenharia da Universidade Federal de Minas Gerais. Os objetivos do ensaio incluíam a validação do modelo matemático de determinação da temperatura superficial da conexão na amostra, bem como do perfil térmico dos cabos em regime permanente. $\mathrm{O}$ esquema básico da montagem utilizada está representado na Figura 5.

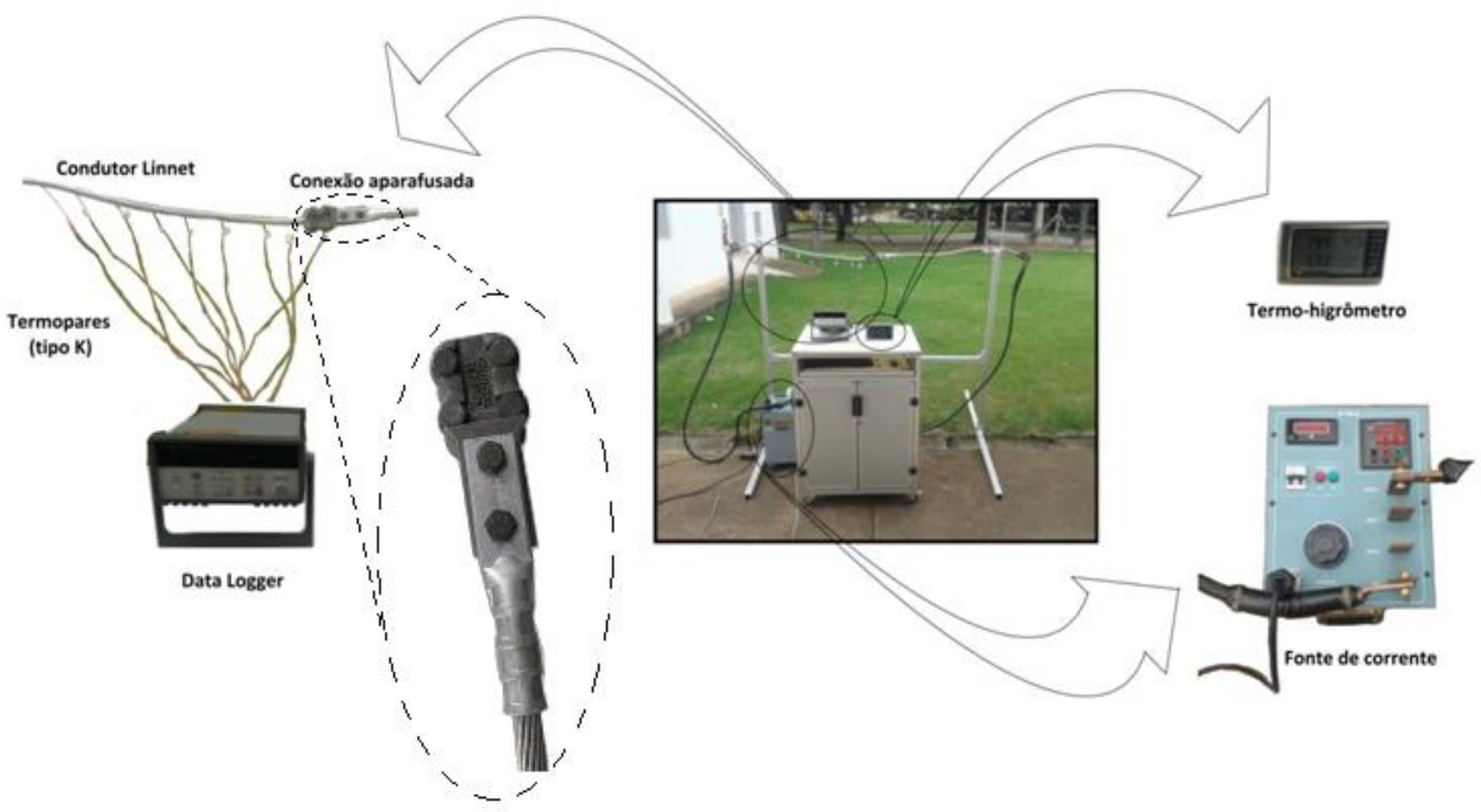

Figura 5. Montagem do ensaio.

O ensaio consistia em energizar o circuito através de uma fonte de corrente e registrar a evolução com o tempo das temperaturas na superfície do conector sob análise, bem como da temperatura ambiente. Para esse fim, um conjunto de 10 termopares tipo "K" foi utilizado, sendo um afixado na superfície do conector, um para tomada de temperatura ambiente e os demais igualmente espaçados entre si ao longo do cabo, conforme a Tabela 1. 
Tabela 1. Posição dos termopares.

\begin{tabular}{cccc}
\hline Canal & Posição & Canal & Posição \\
\hline 1 & - & 6 & Cabo $(x=39 \mathrm{~cm})$ \\
2 & Conector & 7 & Cabo $(x=52 \mathrm{~cm})$ \\
3 & Cabo $(x=0 \mathrm{~cm})$ & 8 & Cabo $(x=65 \mathrm{~cm})$ \\
4 & Cabo $(x=13 \mathrm{~cm})$ & 9 & Cabo $(x=78 \mathrm{~cm})$ \\
5 & Cabo $(x=26 \mathrm{~cm})$ & 10 & Cabo $(x=91 \mathrm{~cm})$
\end{tabular}

Uma amostra de 2,3 $\mathrm{m}$ de cabo foi selecionada para esse tipo de teste, juntamente com um conector tipo aparafusado. Após as devidas precauções para isolamento do circuito, de forma a se evitar fuga de corrente ao longo da estrutura metálica de sustentação, a fonte de corrente foi ajustada para $400 \mathrm{~A}$ enquanto um Data Logger registrava e enviava o sinal de temperatura para um computador, sendo que cada canal transmitia os dados a uma taxa de 1:10 (uma medição a cada 10 segundos).

\section{RESULTADOS E DISCUSSÃO}

No Gráfico 1 pode ser observado o comportamento térmico do conector durante as 4,5 $h$ de ensaio e também a variação da temperatura ambiente registrada no respectivo intervalo do dia. Para os $400 \mathrm{~A}$ aplicados, a temperatura em regime permanente da amostra ficou próxima aos $70^{\circ} \mathrm{C}$. A tendência de aumento da temperatura do conector com o aumento da temperatura ambiente é verificada e as oscilações observadas podem ser atribuídas à variação natural do valor de corrente fornecido pela fonte.

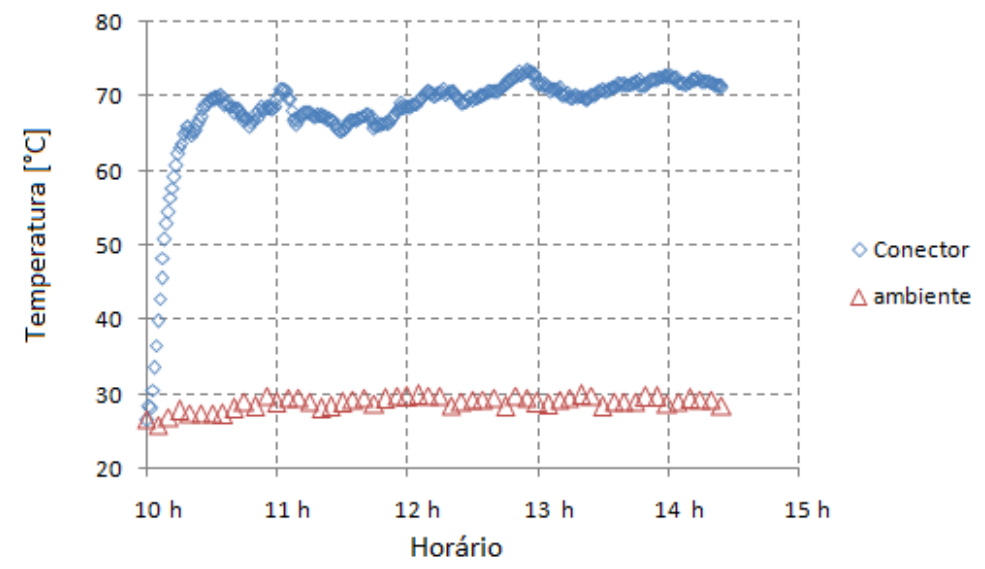

Gráfico 1. Evolução da temperatura superficial do conector durante o ensaio.

Para análise do efeito aleta nos cabos, foi selecionado o instante de tempo em que o conector apresentou temperatura superficial de $70^{\circ} \mathrm{C}$ e foi assumido o completo estabelecimento do regime permanente nesse momento. $\mathrm{O}$ Gráfico 2 apresenta o perfil de temperaturas registrado experimentalmente em comparação com as curvas teóricas obtidas através das soluções numérica e analítica da equação diferencial associada. Também é apresentada a solução exata para o caso da aleta sem geração de calor (Dewitt e Incropera, 2014).

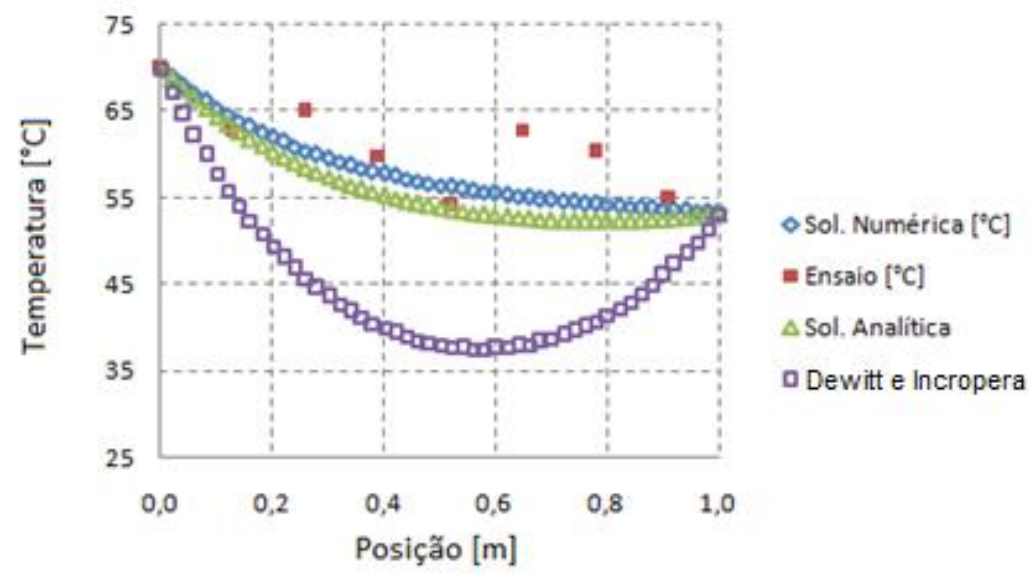

Gráfico 2. Comparação entre os perfis de temperatura teórico e experimental. 
Observa-se pelo gráfico que a solução analítica para o caso de aleta sem geração de calor claramente se afasta da situação real e, portanto, não se aplica ao problema em questão. Ao comparar as curvas teóricas restantes com os pontos experimentais, salienta-se que a precisão destes dados sofre alterações de canal para canal, uma vez que cada termopar foi individualmente fixado e esse fato inevitavelmente gera desvios na leitura. A menos dessa consideração, os erros entre modelo e experimento em termos de temperatura foram inferiores a $15 \%$.

Uma estimativa inicial da taxa de transferência de calor pode ser extraída do experimento, tomando como referência os dois primeiros canais de termopar, posicionados entre si a uma distância conhecida, e aplicar a Lei de Fourier:

$$
q_{\text {cond }}=-k A_{s r} \frac{d T}{d x} \cong-k A_{s r} \frac{\Delta T}{\Delta x}
$$

Logo:

$$
q_{\text {cond }}=-250 \frac{W}{m K} 198 \times 10^{-6} m^{2} \frac{(62,73-70,0)^{\circ} \mathrm{C}}{0,13 m} \times \frac{1 K}{1^{\circ} \mathrm{C}}=2,77 \boldsymbol{W} \text { (Experimental) }
$$

O conector utilizado estava em bom estado de conservação e com correta aplicação de carga nos parafusos. Considerando que o mesmo apresentava uma resistência típica de junções aparafusadas novas $(100 \mu \Omega)$, a passagem de $400 \mathrm{~A}$ de corrente garantia uma geração de calor no interior da conexão de $16 \mathrm{~W}$. Ressaltando que a geometria do conjunto é composta por duas aletas simétricas, esses valores calculados indicam que aproximadamente 35\% do calor gerado no interior do conector foi dissipado por condução axial para os cabos e o restante dissipado através dos mecanismos de convecção e radiação.

Os valores teóricos para as taxas de transferência de calor por condução foram calculados segundo os métodos numérico e analítico, sendo apresentados a seguir:

\section{$\Rightarrow$ Solução numérica:}

$\begin{array}{lll}\circ & \text { Lei de Fourier: } & q_{\text {cond }}=2,69 \mathrm{~W} \\ \circ & \text { Balanço global: } & q_{\text {cond }}=2,55 \mathrm{~W} \\ \circ & \text { Método das somas: } & q_{\text {cond }}=2,50 \mathrm{~W}\end{array}$

\section{$\Rightarrow$ Solucão analítica:}

$$
\begin{array}{lll}
\circ & \text { Com geração de calor: } \quad q_{\text {cond }}=3,39 \mathrm{~W} \\
\circ & \text { Sem geração de calor (Dewitt e Incropera): } \quad q_{\text {cond }}=6,60 \mathrm{~W}
\end{array}
$$

Diferente da solução analítica, a solução numérica não limita o problema a parâmetros constantes, permitindo variações locais da geração de calor, bem como dos coeficientes convectivos e de radiação. Para a obtenção da solução analítica, tal liberdade foi restringida sendo que foi necessário assumir um valor para o coeficiente total de dissipação $\left(h_{T}\right)$, constante para todo o cabo. Torna-se necessário, portanto avaliar os efeitos da estimativa imprecisa desse parâmetro no modelo e como isso pode interferir no perfil térmico e no cálculo da taxa de transferência de calor por condução na base da aleta.

As curvas apresentadas no Gráfico 3 são os perfis térmicos obtidos com a solução analítica simulando estimativas imprecisas do coeficiente total de dissipação (por convecção e radiação), com valores 10\%, 20\% e 30\% abaixo e acima do valor tomado como verdadeiro.

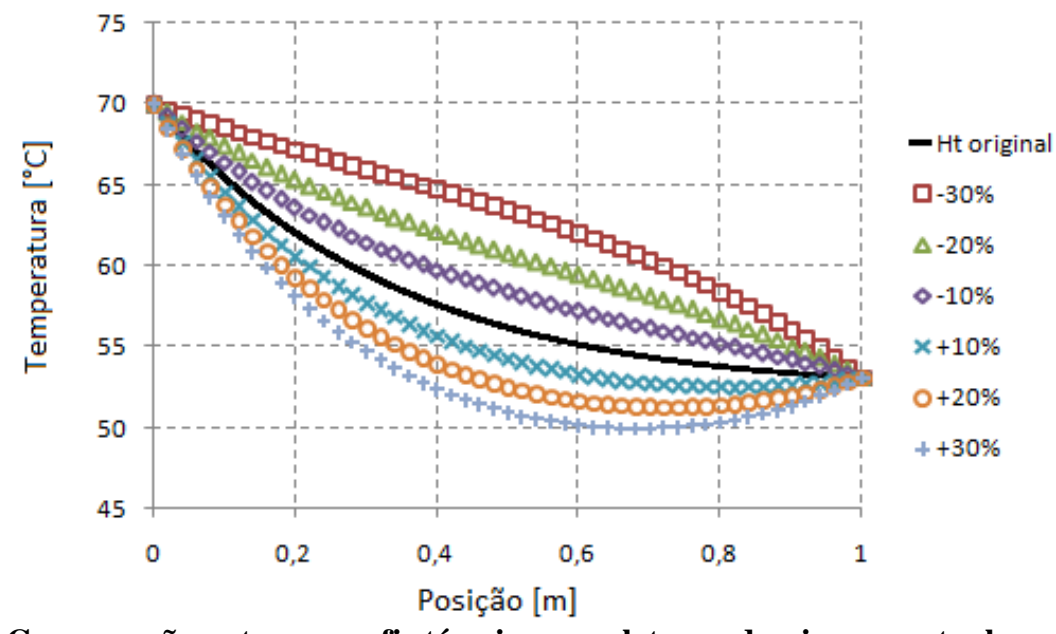

GRÁFICO 3. Comparação entre os perfis térmicos na aleta e o desvio percentual no coeficiente $h_{T}$. 
Nesta simulação, a utilização de valores de $h_{T}$ menores implica na subestimação da capacidade de dissipação de calor pelos mecanismos de convecção e radiação e, consequentemente, um aumento na temperatura média da aleta. A análise para valores maiores da constante $h_{T}$ é similar. Comparando os casos extremos, as curvas $h_{T}+30 \%$ e $h_{T}-$ $30 \%$ apresentaram erros máximos de $5,30^{\circ} \mathrm{C}$ e $7,25^{\circ} \mathrm{C}$ respectivamente, mostrando ser mais crítica a situação em que esse coeficiente é subestimado. Essa conclusão também pode ser observada a partir da Tabela 2, onde estão reunidas as taxas de transferência de calor calculadas para cada condição simulada.

Tabela 2. Taxas de transferência de calor calculadas.

\begin{tabular}{ccc}
\hline Coef. de dissipação & $\boldsymbol{q}_{\text {cond }}[\boldsymbol{W}]$ & Desvio \\
\hline$h_{T}-30 \%$ & 0,78 & $-69,9 \%$ \\
$h_{T}-20 \%$ & 1,43 & $-44,8 \%$ \\
$h_{T}-10 \%$ & 2,03 & $-21,6 \%$ \\
$h_{T}$ & 2,59 & - \\
$h_{T}+30 \%$ & 3,11 & $20,1 \%$ \\
$h_{T}+20 \%$ & 3,60 & $39,0 \%$ \\
$h_{T}+10 \%$ & 4,06 & $56,8 \%$
\end{tabular}

Conforme mencionado anteriormente, o método do fracionamento do domínio físico permite calcular individualmente as taxas de transferência de calor em cada elemento criado e, portanto, observar graficamente como esses valores se apresentam ao longo da aleta como um todo. Para tanto foi definido o parâmetro $\Delta q_{\text {cond }}-$ a diferença entre a taxa de transferência de calor por condução que entra e que sai de cada elemento e que, pelo balanço de energia (em regime permanente), deve se equilibrar com a geração e dissipação. O Gráfico 4 ilustra a resposta desse modelo, simulando as condições de ensaio submetidas à amostra durante o experimento.

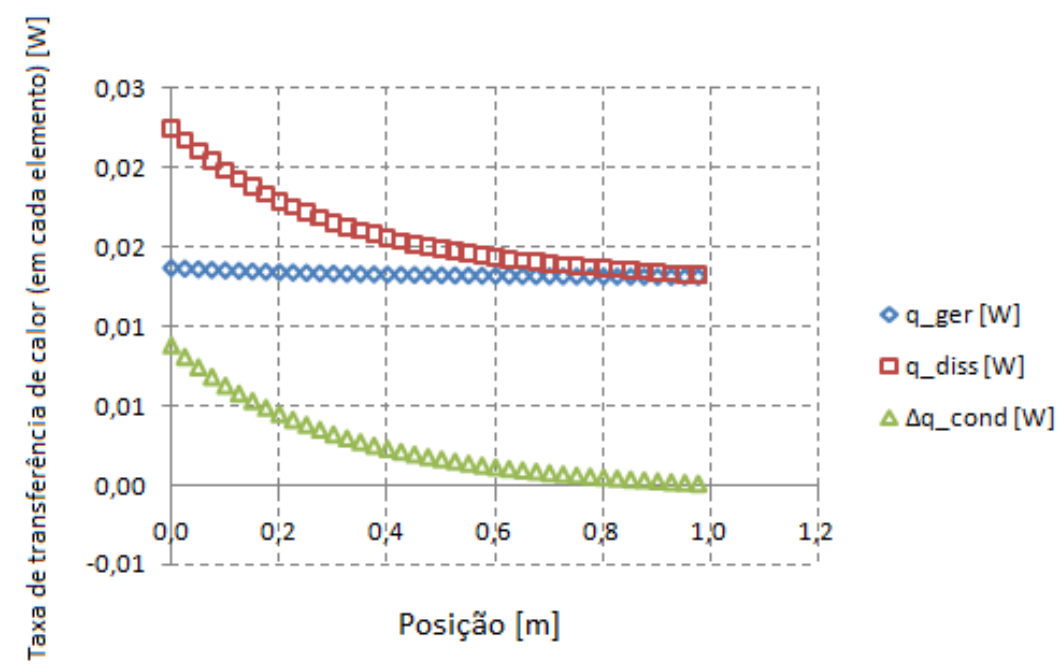

Gráfico 4. Representação das taxas de transferência de calor atuantes em cada elemento gerado ao longo do comprimento da aleta discretizada. $(\Delta x=0,001 \mathrm{~m})$.

A magnitude dos valores representados nesse gráfico naturalmente depende do tamanho definido para os elementos, mas as tendências observadas se repetem para outras escolhas de $\Delta x$. Esse gráfico mostra que a suposição de geração de calor uniforme ao longo da aleta é uma suposição razoável, mesmo que a geração seja ligeiramente maior nas proximidades do conector. A dissipação combinada dos mecanismos de convecção e radiação decai monotonicamente ao longo da aleta até se igualar ao valor da geração, já que a partir desse ponto são desprezíveis os efeitos de condução axial e, em conseqüência disso, a curva da taxa $\Delta q_{\text {cond }}$ tende a zero.

Por fim, um gráfico desse tipo também pode fornecer uma informação interessante a respeito de um comprimento crítico, a partir do qual a temperatura do cabo passa a não sofrer interferência devido a proximidade de um conector sobreaquecido. No caso específico dessa análise, esse comprimento é de aproximadamente $80 \mathrm{~cm}$. Um dado desse tipo pode ser útil na emissão de diagnóstico de cabos, garantindo que a temperatura medida não está sendo mascarada por nenhum efeito de proximidade. 


\title{
5. CONCLUSÕES
}

Ao modelar o processo de aquecimento de conexões elétricas, os resultados experimentais mostram que a taxa de transferência de calor por condução é, em geral, significativa. A comparação entre as abordagens numérica e analítica de solução da equação diferencial associada ao perfil de temperaturas mostra que a primeira maneira é mais indicada, uma vez que sua formulação admite variações locais das propriedades físicas e, por outro lado, a solução analítica por coeficientes constantes depende fortemente da precisão na determinação dos valores médios desses parâmetros. Dentre outras vantagens, a solução numérica permite o cálculo de um comprimento de referência, a partir do qual os valores de temperatura medidos não são influenciados pela proximidade com um ponto de anomalia térmica, uma informação extremamente útil para as equipes de manutenção de componentes elétricos desse tipo, auxiliando no diagnóstico e no processo de tomada de decisão.

\section{AGRADECIMENTOS}

Os autores agradecem aos financiadores do projeto P\&D 426, ANEEL-CEMIG, e à equipe do LabTerm pela disponibilização dos equipamentos necessários para a condução desse trabalho.

\section{REFERÊNCIAS}

Bejan, Adrian; Transferência de Calor. ed., Edgard Blücher Ltda, 1996.

Braunovic, Milenko; Electrical Contacts: Fundamentals, Applications and Technology. Taylor \& Francis Group, LLC. New York, 2006.

Çengel, Yunus A.; Transferência de Calor e Massa: uma abordagem prática. 3.ed.McGraw-Hill, São Paulo, 2009.

Dewitt, David P.; Incropera, Frank P. Fundamentos de Transferência de Calor e Massa. 7.ed.LTC, 2014.

Diniz, H.E.P. Termografia Quantitativa como Ferramenta de Gestão de Ativos do Sistema Elétrico de Potência. Dissertação de Mestrado.Universidade Federal de Minas Gerais. Belo Horizonte, 2013.

Rocha, V.L et al. Cálculo Numérico - Aspectos Teóricos e Computacionais. $1^{a}$ ed., McGraw-Hill, São Paulo, 1988.

Santos, Reginaldo J. Introdução as Equações Diferenciais Ordinárias. Belo Horizonte, Imprensa Universitária da UFMG, 2011.

Stewart, James; Cálculo, volume I. 7.ed. Pioneira Thomson Learning. São Paulo, 2013.

\section{RESPONSABILIDADE AUTORAL}

\section{MATHEMATICAL MODELING OF EFFECT OF THERMAL CONDUCTION IN HEATING PROCESS OF CONDUCTING CABLES}

\author{
Rafael Augusto Magalhães Ferreira, r.ferreira102@ hotmail.com ${ }^{1}$ \\ Roberto Márcio de Andrade, roberto@demec.ufmg.br ${ }^{2}$ \\ ${ }^{1}$ Universidade Federal de Minas Gerais - Departamento de Engenharia Mecânica - Av. Antônio Carlos 6627, Belo \\ Horizonte - MG, Brasil. \\ ${ }^{2}$ Universidade Federal de Minas Gerais - Departamento de Engenharia Mecânica - Av. Antônio Carlos 6627, Belo \\ Horizonte - MG, Brasil.
}

\begin{abstract}
Cable conductors are essential elements for the electric power system, in particular for the power distribution system. In substations, due to the amount of equipment and subsystems, many energized lines are sectioned and connected on many points, presenting a natural temperature increase in these regions due to heat generation by Joule effect. The temperature difference between the region of electrical connection and cables generates a portion of heat transfer by conduction, which can be modeled from an ordinary differential equation of second order, inhomogeneous and nonlinear. This work aims to confront the numerical and analytical solutions of the problem, assess the adequacy of the simplifications assumed for the physical phenomenon and perform experimental validation through testing current. The results show a good approximation between model and experiment, and from the point of view of monitoring conductor cables, this modeling is useful in identifying the distance from the point of heating that is necessary for the measurement of temperature in the cable is not influenced by the effects of axial heat conduction; contributing to an increase in reliability of thermal records and thus the diagnosis of the component.
\end{abstract}

Keywords: electrical connectors, mathematical modeling, finned systems, differencial equations discretization.

Os autores são os únicos responsáveis pelo conteúdo deste trabalho. 